\title{
A High Speed Networked Signal Processing Platform for Multi-element Radio Telescopes
}

\author{
Peeyush Prasad · C.R. Subrahmanya
}

the date of receipt and acceptance should be inserted later

\begin{abstract}
A new architecture is presented for a Networked Signal Processing System (NSPS) suitable for handling the real-time signal processing of multi-element radio telescopes. In this system, a multi-element radio telescope is viewed as an application of a multi-sensor, data fusion problem which can be decomposed into a general set of computing and network components for which a practical and scalable architecture is enabled by current technology. The need for such a system arose in the context of an ongoing program for reconfiguring the Ooty Radio Telescope (ORT) as a programmable 264-element array, which will enable several new observing capabilities for large scale surveys on this mature telescope. For this application, it is necessary to manage, route and combine large volumes of data whose real-time collation requires large I/O bandwidths to be sustained. Since these are general requirements of many multi-sensor fusion applications, we first describe the basic architecture of the NSPS in terms of a Fusion Tree before elaborating on its application for the ORT. The paper addresses issues relating to high speed distributed data acquisition, Field Programmable Gate Array (FPGA) based peer-to-peer networks supporting significant on-the fly processing while routing, and providing a last mile interface to a typical commodity network like Gigabit Ethernet. The system is fundamentally a pair of two co-operative networks, among which one is part of a commodity high performance computer cluster and the other is based on Commercial-Off The-Shelf (COTS) technology with support from software/firmware components in the public domain.
\end{abstract}

Peeyush Prasad

Astronomy and Astrophysics Group,

Raman Research Institute,

Bangalore - 560 080, India

Tel: $+91-80-23610122$

Fax: $+91-80-23610492$

E-mail: peeyush@rri.res.in

C.R. Subrahmanya

Astronomy and Astrophysics Group,

Raman Research Institute,

Bangalore - 560 080, India

Tel: +91-80-23610122

Fax: +91-80-23610492

E-mail: crs@rri.res.in 
Keywords Radio Interferometry · Multibeam Phased Array · FPGA · Signal Processing $\cdot$ High speed networks · Distributed real-time processing.

\section{Introduction}

A Multi-element Radio Telescope is a spatially spread array of antennas (or antenna elements) whose noise-like responses are required to be time aligned, dynamically calibrated and combined or correlated in real time. The resulting estimates of spatiotemporal and spectral correlations between responses of pairs of elements can be used to recover the desired information on the strength and distribution of radio emission within the common field of view (Thompson et. al., 1994) using standard postprocessing software. Thus the signal of interest is statistical in nature, resulting from a minute level of mutual coherence arising from weak celestial signals buried in noise. Because of the large number of elements and the high sampling rates necessary for bandwidths exceeding several tens of $\mathrm{MHz}$ in recent arrays, real-time statistical estimation is essential to achieve practical data rates and volumes for recording and post processing. For instance, the recently initiated upgrade for the Ooty Radio Telescope (ORT) aims at treating the $30 \mathrm{~m} \times 506 \mathrm{~m}$ parabolic cylindrical antenna of the ORT as 264 independent sets of elements, each of which is required to be sampled at $80 \mathrm{Ms} / \mathrm{s}$, leading to a data generation rate of 21 Gigasamples per second, exceeding 80 Terabytes per hour. Till recently, computing requirements of this scale forced a choice of custom hardware to be the most favored platform. However, rapid developments in the fields of digital technology, communication and computing have led to a changing trend towards alternative approaches for upcoming telescopes. Such approaches range between a customized and reusable hardware library of components on an FPGA platform, e.g., the CASPER project (Parsons et. al, 2009), and a software-only approach, e.g., at the GMRT (Roy et. al, 2010). The GMRT case is an example of a recent transition from custom hardware to a software-only approach.

In this paper, we have taken a middle path, where the real-time processing of a multi-element radio telescope is abstracted as a multi-sensor, data fusion problem and addressed in a new platform called the Networked Signal Processing System (NSPS) in terms of packetized, heterogeneous, distributed and real-time signal processing. It is a co-operative of two kinds of networks, among which one is a custom peer-topeer network while the other is a part of a commodity processor network. The custom network includes subsystems related to the digitization and all intermediate routing and preprocessing blocks as the network nodes, in which the emphasis is on traffic shaping, on-the-fly processing and load balancing for effective distributed computing. However, all customized protocols are absorbed while crossing over the last mile to interface to the commodity processor network using a common industry-standard network protocol. The actual estimation of the correlations is carried out by nodes on the commodity network.

In contrast to the traditional use of a packetized network as merely a data transport fabric between processing entities, we have the notion of "a logical packet" based on an application specific "transaction unit", which itself may be composed of a large number of physical packets whose sizes are network-specific This unit refers to a time stretch long enough to facilitate a dynamical flagging mechanism or/and to relax the constraint on timing, synchronization and scheduling of workloads on the commodity Operating Systems on which processing is expected to be carried out. Both these requirements 
necessitate lower level NSPS nodes to be equipped with large memories, which are also used to route traffic selectively (traffic shaping) to higher levels in the NSPS. Two independent considerations have led us towards stretching the transaction unit to a good fraction of a second. One of these, as explained above, is to provide latency tolerance in order to simplify software on standard computing platforms, while the other arises from a desire to make explicit provision for preprocessing using concepts related to modern Information Theory. From this point of view and to attract the attention of experts from outside the field of radio astronomy, we have given a somewhat unconventional description of the signal path and analysis of processing requirements in Sections 2 and 3 in an attempt to illustrate the connection of the problem to Information Theory.

Another concept in literature which we find useful in the present context is that of "multi-sensor data fusion", defined by (Klein, 2004), as a system model where "spatially and temporally indexed data provided by different sources are combined (fused) in order to improve the processing and interpretation of these data". This model, widely used in applications like military target tracking, weather forecasting etc, has many features relevant for describing the control flow and pre-processing required in a multi-element radio telescope before correlation. In a sense, the NSPS is an adaptation of data fusion architecture to our domain. Our analysis of the nature of the real-time problem results in a natural partitioning into two broad categories as elaborated in Section 3 This is our primary motivation for defining the architecture, described as a Fusion Tree in Section 4. An illustration of the feasibility of its implementation is presented in Section 5 by considering the case of the ORT upgrade.

\section{A Multi-Element Radio Telescope as a Set of Correlated, Noisy Carriers of Information}

We present here an abstract picture of a multi-element radio telescope, in which the primary beams of individual elements are viewed as virtual communication channels carrying different combinations of radiation from a set of independent celestial "radio emitters". These are located in different directions within the common celestial region intercepted by the element primary beams. Each individual radio emitter from this set is a source of stationary (within the observing time) random process. Since signals received in any finite bandwidth can be spectrally decomposed, each primary beam can be considered equivalent to a set of independent communication channels of identical bandwidths corresponding to the spectral resolution. Each such channel is characterized by a correlation timescale equal to reciprocal of its bandwidth. Thus, spectral decomposition can be used to enable the propagation time differences for noise from different radio emitters to be within their correlation timescale. This implies that the corresponding channels of different primary beams are carriers of noise arising from different superpositions of the same set of random processes, but with different weights. Information about the strength and distribution of radio emitters in the field of view can be considered to be coded in the correlation between corresponding communication channels of different elements. Hence, we consider the real-time spectral correlation to be a fusion process for compressing the information conveyed by the responses of different elements without affecting decoding to be carried out in post-processing, say in the form of an image of a celestial region.

However, in practice, the propagation medium introduces a variety of correlated and uncorrelated noise into these virtual channels which can erase or distort the in- 
formation related to celestial emitters. This is represented schematically in Fig. 1] Often, some of these distortions are characterized by a combination of dispersive and non-dispersive components which are localized in time and/or frequency, unlike the celestial signal which is more like a random noise. Such a localization can be exploited by a pre-processing algorithm to enable suitable recognition and characterization of deviant (non-random) data and to tag them suitably. Such data can then be segregated from those passed on to an irreversible fusion operation, e.g., to minimize the biases in the correlations. On the other hand, in situations like observing fast transients with low duty cycle, or when one wants to find the direction in which such a non-random signal is present, the segregated data can be passed to an independent processing or recording stage for later use. We refer the interested reader for an analogous situation in a re-visit of Maxwell's demon by (Zurek, 1989) to connect algorithmic randomness to physical entropy. For the present purpose, it suffices to note that such a segregation results effectively from an algorithmic feedback, requiring multiple passes through the data before fusion. The output of this feedback via multiple passes on stored data is also analogous to the concept of a relay network with "side" or meta information, described in (Cover and Thomas, 2006), where this side information is used by other entities for selective processing of the data reaching them. In our abstraction, a provision for such an algorithmic feedback should be an essential part of the signal processing architecture, and should be present in the path between the digitizer and the fusion operator. This is only possible if the real-time system is equipped with adequate memory and preprocessing for characterization of data before they are sent to a correlator or beam forming system. By excluding such a provision, digital receivers in existing large radio telescopes are a potential source of irreversible biases in the recorded correlations, and suffer from inefficiency for requirements like detection of short term transients.

\section{Real-Time Processing Requirements}

Without getting into specific details of the real-time processing required for a large multi-element radio telescope, we abstract them into a combination of three broad categories:

- Embarrassingly Parallel processing, e.g., spectral decomposition of the incoming time series (say via FFT or polyphase filter banks) and the recognition and management of path-induced distortions/interference on timescales significantly smaller than the integration time chosen for the correlations.

- Pipelined processing, e.g., multi-beam formation (say $K$ beams) by phasing $\mathrm{N}$ elements requires $K \log _{2} N$ pipelined operations for each spectral band.

- Data Fusion operations, in which the data originating from different antenna elements are hierarchically fused (combined via routing and application-dependent processing) along a chosen set of dimensions which include time, frequency and spatial spread.

The most important fusion operation for an antenna array is the real-time correlation of signals from every possible pair of antenna elements in different frequency sub-bands. Apart from being an $O\left(N^{2}\right)$ process (for an $\mathrm{N}$ element array) from a computational point of view, this brings in the additional complication of routing large volumes of distributed data to appropriate data processing elements to provide a complete-graph connectivity between the sources of data and processing elements. Significantly, crosscorrelation between all possible pairs of signals is also essential for using self-calibration 


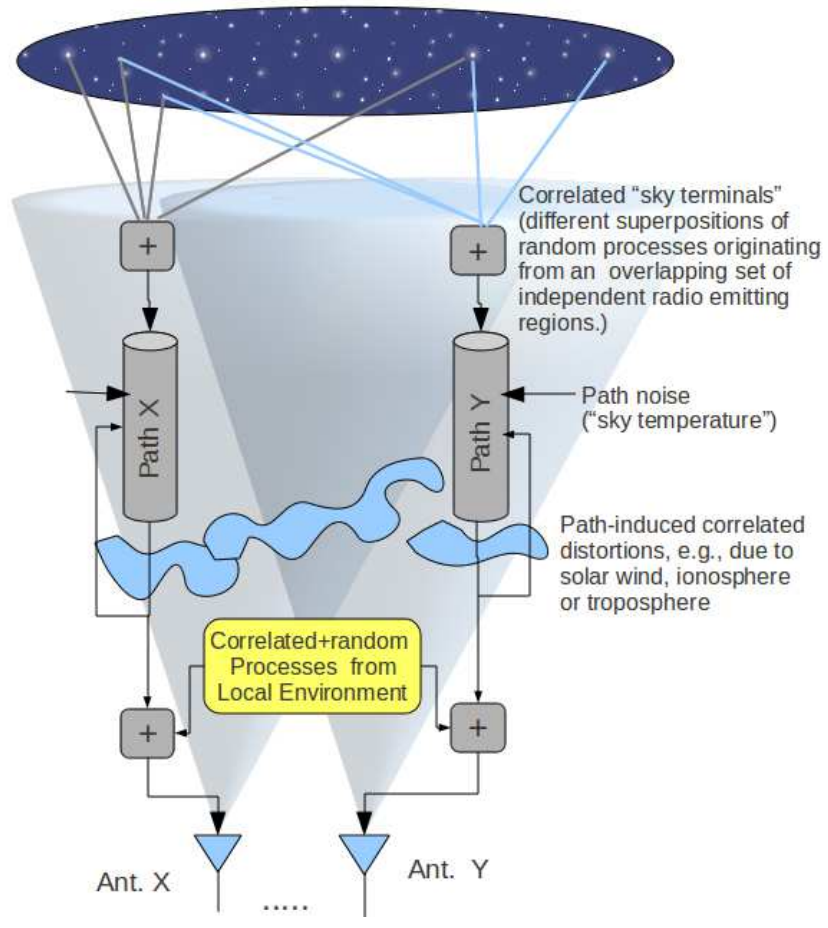

Fig. 1 Antenna beams viewed as noisy virtual communication channels connecting a sky region and an antenna element.

techniques to enable dynamic calibration of instrumental and atmospheric contributions to the data corresponding to different elements, before they are subjected to an irreversible fusing operation in a phased array. This makes a spectral correlator an implicit requirement, even for a phased array, for minimizing the irreversible loss of information resulting from distortions induced in the the path or the local environment.

In our approach, we bifurcate the requirements of a real-time system into Commodity and Custom segments. In the current state of technology, the commodity segment 
can be fulfilled by subsystems available in the market while the custom segment can generally be realized on the basis of customized hardware and/or firmware layers based on COTS technology. Such a bifurcation is explained below for different functional categories of the NSPS:

\subsection{Computation:}

- COTS Segment: Computationally complex and/or latency tolerant processing, typically realized on a programmable platform ranging from workstations to a high performance cluster.

- Custom Segment: Latency critical, logic intensive and repetitive pattern of deterministic processing, well suited for a configurable platform, typically FPGA-based.

For efficient computation, we pay special attention to reducing coupling between data in order to target explicit parallelism at all levels of processing. Multiple parallel circuits are implemented in the FPGA-based custom segment, while the current trend of multicore processors with access to shared memory is exploited in high level software. Further, the desired high signal bandwidth and large number of antenna elements make the processing complex and compute intensive. This aspect, and the advantage of quickly implementing exploratory algorithms, make a commodity compute cluster an attractive choice for the central computing. This is recognized by explicitly including the cluster in the COTS segment mentioned above.

\subsection{Data Routing:}

- COTS Segment: Commercial switches with all-to-all connectivity are used for data routing to commodity processors and broadcasting in the last mile, as well as for load balancing. The routing is controlled by manipulating the destination addresses on data packets. Connection-less protocols like User Datagram Protocol (UDP) are adequate for high-speed, streaming applications where a small fraction of lost packets does not affect performance adversely. Packet collisions are minimized in full duplex, point-to-point connections between network partners, and also because data flow is extremely asymmetric. Further, the criteria for load partitioning discussed in Section 4.8 very often result in under-utilization of link speeds to match them to sustainable processing bandwidths.

- Custom Segment: Customized switches with static routes for traffic shaping are relevant when only a subset of the network data needs to flow to a subset of the nodes based on certain conditions. They are generally implemented in configurable logic.

Since many FPGAs support Gigabit Ethernet MAC as a hard (or publicly accessible soft) IP, this feature is useful while introducing a bridge between the peer-to-peer network and the commodity network in the last stage of the custom segment. In addition, some or all the major subsystems may have management support from an embedded or explicit on-board processor. 


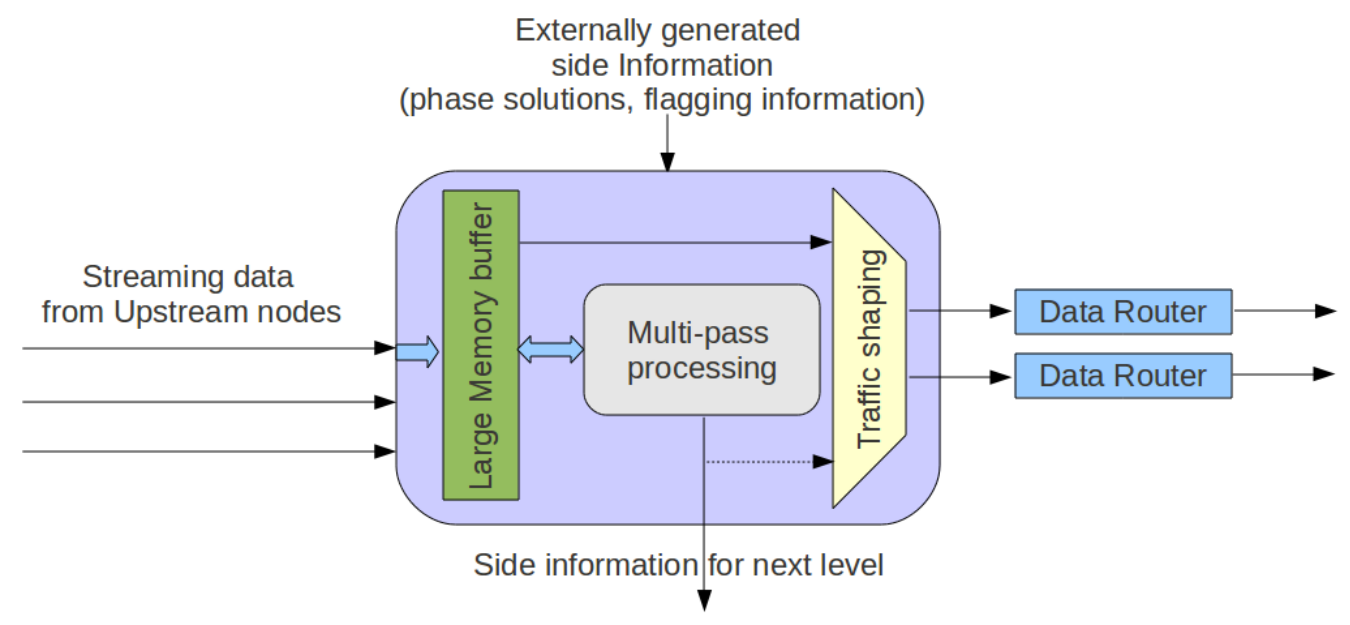

Fig. 2 A Data Pooler node of the NSPS shown carrying out data fusion and traffic shaping.

3.3 Network Topology and Protocol:

- COTS Segment: A commodity network compatible with a typical high performance compute cluster, which includes Gigabit Ethernet as a de facto standard for interfacing with external systems.

- Custom Segment: A peer-to-peer network which may include significant on-thefly application specific operations, suitable for implementing on a standard FPGA platform.

An implementation of the actual processing on a dedicated set of identical hardware circuits or parallel processors can take advantage of an intelligent network capable of elementary on-the-fly operations to achieve a balance on the dataflow and computing requirements. This is depicted in Fig. 2] where a "Data Pooler" node, as defined in Section 4, is shown carrying out real-time fusion of the streaming data by using the side information made available by external sources. At the same time, the pooler is seen generating side information out of the fused data set by way of multiple processing passes on the stored data. The pooler can then segregate the data, and route the segregated components to different data sinks using the routing information available with the peer-to-peer link nodes. We use "traffic shaping" here in a more general sense than in Internet traffic shaping (which delays lower priority packets in favour of better network performance of higher priority packets) to refer to both segregation of the incoming stream, as well as the specific routing of segregated data to different sinks. Further, the efficiency of hierarchical computation can be significantly improved by accommodating some degree of pre-processing and/or partitioning of data in each level of the custom segment to facilitate the next level.

\subsection{Process Scheduling:}

- COTS Segment: The overall task supervision, command and monitor, user interface and the dynamic system monitoring are tasks whose complexity is best left to the 
commodity segment to handle, where a variety of tools ranging from MPI, compiler resources and advanced operating systems like Linux or VxWorks are available.

- Custom Segment: Event driven scheduling with periodic or quasi-periodic events generated conveniently in a low latency logic implementation suitable for an FPGA. The interval between the events is stretched to handle an application-specific transaction unit to the extent permissible within the available resources.

\section{NSPS as a Fusion Tree}

In this Section, we present the NSPS architecture as a Multi-sensor Data Fusion Tree, in which both conventional and "virtual" sensors play a role. While entities like antenna elements, round-trip phase/delay monitors, noise calibration etc can be treated as "conventional" sensors, "virtual" sensors result from processing blocks at various levels. For instance, pre-processing can result in a flagging mechanism to improve the reliability of fusion systems like correlators, in which the original data are erased while compressing their information into a statistical estimate to be passed to the next level.

The signal processing system proposed in this paper is a set of spatially separated nodes of varying communication and processing capabilities, which are interconnected by a customized high speed tree-like packet switched network interfaced to master commodity nodes. This is equivalent to a Data Fusion Tree, with the nodes of the tree performing operations like traffic shaping, packet routing, or pre-processing before data fusion. Accordingly, we have described the overall architecture of NSPS in the form of a fusion tree, schematically represented in Fig. 3 However, each level provides a different mixture of functional capabilities. This has resulted from our recognition of the following features of the functional requirements:

- Distributed computing across many nodes of different processing capabilities, with local parameters guided by a processor capable of seeing a subset of all data.

- Data routing nodes with configurable routes for routing preprocessed data subsets.

- Nodes with large memory buffers to enable multiple passes on data, also for enabling memory based data transposition.

- A High speed network interconnecting all nodes, with ability of COTS nodes to tap into the network.

- Interface to a master commodity node through a standard interface like Gigabit Ethernet (GigE).

Thus, from a functional point of view, we classify the nodes in NSPS into the following three categories:

1. Data Poolers/Fusers: Nodes with sufficient on-board memory to allow packetizing and multiple processing passes on incoming data. These break the need for manyto-many connectivity in the correlation process by transposing data via memory based switches. The transposition, based on different parameters, ultimately serves a packet of data suitable for processing by a single element of a distributed system in an embarrassingly parallel manner.

2. Data Routers: These elements are endowed with high speed links to either peers or more powerful processors to whom incoming packets are routed based on statically configured routes. These form an integral part of our architecture, helping in the load balancing by directing appropriate subsets of preprocessed data to different 
processing elements. We use commodity switches for routing data to multiple external sinks by forming many-to-many connections between data sources and data processors.

3. Data Processors: These elements have high compute density and can be used for preprocessing, as well as for data rate reduction. We classify processors into two groups as mentioned earlier:

(a) Those catering to computationally complex and/or latency tolerant processing, an example of which is the estimation of system calibration parameters based on a long (few minutes) history of data, and its dynamic updation. This processing is generally carried out by sending a subset of the data to a central processor.

(b) Latency critical, logic intensive and repetitive pattern of deterministic processing. An example of this class is the real-time block-level data encoding process requiring the estimation of block level statistics. This can be carried out by multiple passes on small segments of data.

Thus, we visualize the NSPS as a restricted distributed system, depending primarily on stripped down lightweight networking protocols and the static routes set up during system configuration. Data routers, both customized and commodity, play an important role in reorganizing data to be computationally palatable to processing nodes in this scheme. A master node is in charge of command, configuration and control, and is almost always a commodity node like a PC. It may be noted that custom processing is spread across the NSPS tree by explicitly advocating local intelligence in every node. As an illustration of the inherent facilitation of distributed processing in the NSPS, some important aspects of the interconnection mechanism are elaborated in the following subsections.

\subsection{Fusion tree interconnection model:}

Interconnects in the Data Fusion Tree consist of the following four essential graphs, which can either be logical or explicitly physical manifestations.

1. The Data network is a simplex, high bandwidth net connecting the leaf nodes of the Fusion Tree to a central processor, possibly passing through several collation levels of the signal processing tree.

2. The Control and Monitor network is a full duplex, low bandwidth network and interconnects all nodes hierarchically through management processors to a central monitoring station.

3. The Calib network is a full duplex, low bandwidth network. This allows calibration information to reach the data fusing nodes before the sequence of irreversible fusion operations take place.

4. The Clock network is a full duplex network, providing the distribution of clocking signals to the various nodes, as well as allowing a round trip clock phase measurement.

In actual implementations, it may be simpler to realize these in terms of a set of simplex networks, among which clock, control, monitor and calib are directed towards the leaf nodes while the data and status (including response to monitor queries) belong to simplex networks which flow from different levels of the fusion tree into the master node.

Our network implementation can be bifurcated into the following sets: 


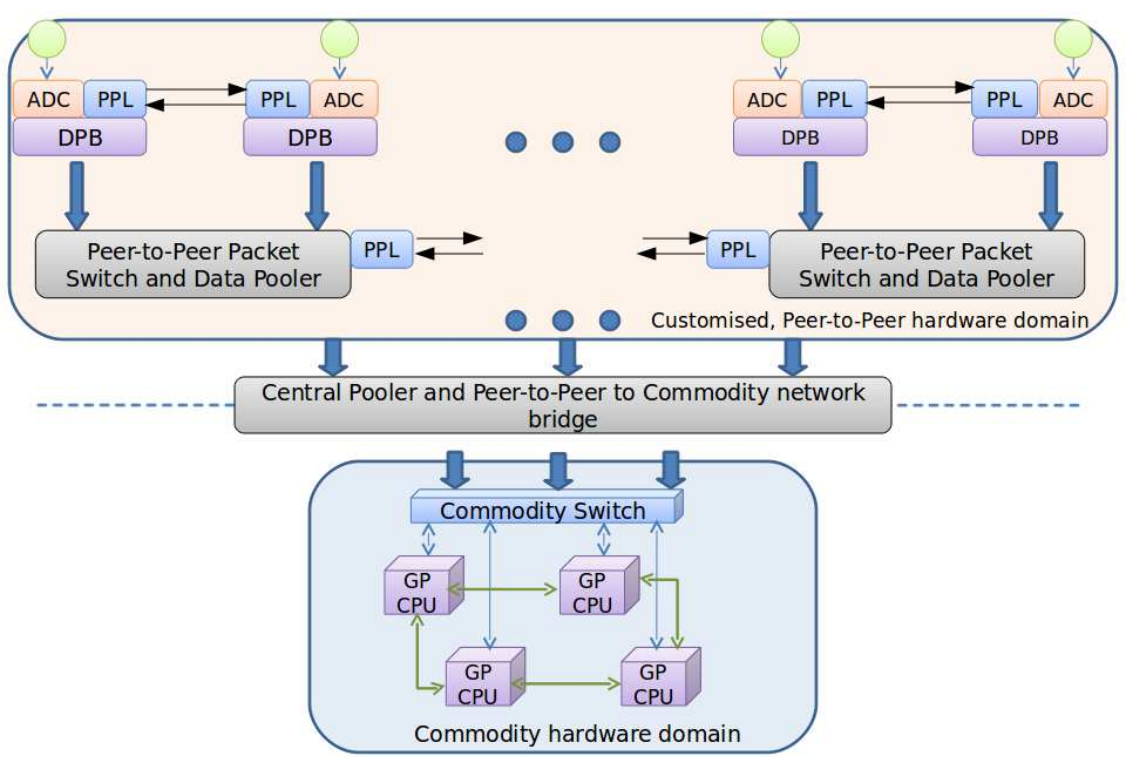

DPB Data Processing Block

PPL Peer-to-Peer Link

Fig. 3 A conceptual layout of a Networked Signal Processing System architecture showing the principal participating entities.

1. Customized high speed serial peer-to-peer links terminating into peer-to-peer switches which implement a subset of the complete graph connectivity.

2. Commodity high speed serial links terminating into commodity networking equipment, with ability to interface to standard processing nodes.

In typical implementations, we expect custom links to be on a Passive Optical Network (PON) based communication stack. Gigabit Ethernet is the preferred choice for the backbone of commodity segment to connect to the custom network.

We exploit the high speed serializing ability of modern FPGAs to dispatch data on high bandwidth copper links and use PON components to meet the spatial spread required to reach remote nodes over fibre links. As long as the bandwidth requirements are met, no specific preference is implied for a choice among different networking technologies. Thus, some implementations may utilize the embedded multi-gigabit serializers in FPGAs for peer-to-peer links while others may refer the cross dispersion of data to a compute cluster's high bandwidth infiniband network, or use commodity Gigabit Ethernet. This leads to the need for a flexible bridging mechanism which can be exploited by implementations. For instance, data can be conveniently transmitted over a peer-to-peer or a commodity link due to the maintained commonality of their interfaces.

4.2 NSPS tree network characteristics:

The high speed network internal to the NSPS tree has features which are restricted and stripped down versions of those found in commodity networks. This is an optimiza- 
tion due to the highly controlled network which exists within the telescope receiver environment. Our network differs from regular networks in several aspects:

- A controller node is assigned for every sub-tree at a given level. This entity forwards command and status information between controller (level-0) and upstream levels. Thus, it is not a typical peer-to-peer network which does not have such a hierarchical control structure. Broadcast and multicast domains available in commodity networks are used to implement control and monitor mechanisms, while explicit "pull" mechanisms are implemented on the custom network. Here, the "pull" refers to the explicit request for data made by a downstream node to an upstream node. The advantage of a "pull" mechanism is that data is made available to a downstream node only when it is ready to handle the data, as inferred from the downstream node's request. Also, if a downstream node is busy, then the upstream node loses data in integral packets, thus maintaining timing information.

- The network configuration and routes are fixed statically in an application dependent manner at configure time. There is no node discovery, and data routing does not have an explicit mechanism for handling node failure. Since all data flows towards a logical sink, there is also no destination address, although source addresses can be preserved. This simplifies network management to a large extent at the cost of non-redundancy of NSPS entities.

- Communication protocols: All elements in our network, including master nodes, generate similar kinds of packets which contain an 8 byte header with fixed fields. These are typically indicative of the nature of accompanying data, as well as its timestamp, source and other meta information. System state can also be propagated through these packets, or by forming special status packets. The restricted meta information processing makes it simpler to realize packet formation in hardware with simple state machines.

- High speed serial interconnects: All entities in the internal network communicate via high speed serial interconnects with a clock recoverable from the encoded data. This approach allows us to transmit data long-haul over fibre, or short-haul over copper without any changes. In particular, we discourage bus-based interconnection between physically separated nodes.

4.3 Interface to external network:

Once preprocessed data is ready within the NSPS, it needs to be transferred onto a commodity network for reaching commodity nodes for post processing or archiving. Local intelligence in the peer network can be used to partition the data such that the interfaces to commodity nodes use link speeds commensurate with their processing ability. For simplicity, we have used Gigabit Ethernet as a typical standard external interface. This is a popular high speed serial interconnect with a vast amount of infrastructure available in the commodity market. It also allows transmission over copper (UTP) to interface directly with commodity servers, or fibre (via conversion to 1000BaseX) for long-haul transmission. Commodity servers of moderate ability can then be used as data sinks with minimal customization. This is also motivated by the fact that many modern FPGAs have embedded high speed serial interconnects on chip, with complete Gigabit Ethernet support in the form of on-chip Gigabit MACs or as publicly available libraries. 
For the last mile connectivity, UDP can be used since it is a simple connectionless protocol with minimal overheads on top of IP. It is also possible to fill the relevant UDP fields during system configuration, and hold them static for the duration of an observation. Each of the internal network types carrying data (Data, Calib and Control) can then be easily made available over a different UDP port as part of the design. This allows an application program to associate independent threads to service these streams.

\subsection{Control and Monitoring network:}

The distributed nature of our architecture requires status monitoring of all nodes and links, which can be handled by the individual sub-tree roots and communicated to the master controller. This is implemented by a status "pull" scheme by which controlling entities periodically query the status of all nodes in the NSPS tree rooted with them by way of a special AYA ("AreYouAlive") packet. The nodes respond with an IAA ("IAmAlive") packet containing selected status information. Similarly, control packets contain command and configuration data. Each command packet typically results in a status reply from the targeted entity, which confirms the receipt of the command, and regenerates a control packet for the entities controlled by it. The master node can use this in an appropriately scheduled housekeeping operation to discover failure of nodes.

\subsection{Calibration network:}

This network is meant to carry data from the NSPS tree which is relevant to forming calibration solutions for the array. The calibration mechanism is to be applied differently for the two main modes of observation with the NSPS:

- In the interferometric mode, the correlator can work independently of the actual gains and phases of the sensor elements, since the observations include calibration scans at reasonable time intervals. Off-line processing can infer intermediate variations by supplementing interpolation between calibration scans with dynamic calibration schemes like self-calibration based on the partial, low bandwidth dataset available over the calibration network.

- On the other hand, real-time beam formation includes an irreversible fusing operation which requires dynamic calibration to be part of data fusion. Fortunately, it is often possible to use a relatively small subset of the data (non-contiguous timeslices or a chosen frequency sub-band) for this purpose to enable short term predictions of gain variations. These can be fed to the fusing nodes well in time before irreversible fusing operations are performed. Since the complexity of the actual algorithm used for calibration makes it better suited for a general purpose computer, the calibration network can be used to route the relevant subset of data to a commodity switch and deliver the calibration parameters to the appropriate NSPS tree level.

4.6 The clock network:

In the spatially distributed, direct RF sampling NSPS architecture, clocks passed to samplers have very stringent signal quality constraints in terms of net jitter and sta- 
bility. The alignment of the multiple data sources before fusion requires high relative stability of the sampling clocks with random jitters much smaller than the reciprocal of the highest frequency in the sampled signal. Clock distribution should also include a mechanism for ensuring the traceability of timekeeping at all digitizing blocks to a centrally maintained time standard to a very high accuracy. The implementation can benefit from commercial clock distributors which have embedded phase-lock loop clock synthesizer with on-chip Voltage Controlled Oscillator (VCO) and a per port delay tuning for the distributed clocks.

\subsection{Data Network:}

All data flowing in the NSPS is packetized with a custom, low overhead header. All subsystems accept and generate data in a packetized fashion. This reflects the inherent asynchronous nature of our system. Packets traversing our platform are atomic and capable of independent existence. The packetizing of data means that data loss due to network congestion or buffer over-runs is never arbitrary, but always in units of packets. At any instant, our network can have different kinds of packets traversing it, corresponding to different stages in the processing. The basic unit of packet size is maintained as 8 bytes, which is a natural unit or sub-unit for different memory and processing hierarchies. Adequate padding is used if necessary to maintain this condition. The header is mandated to have a few fixed fields which are common in size and layout across packet types, allowing processing entities to examine packets which can be processed by them, while discarding the others. In a broadcast network, this approach can waste bandwidth when packets are discarded, but the wastage can be minimised by setting up static routes between partner nodes. This is possible in both the custom and commodity peer-to-peer link nodes. The Command network, on the other hand, is a broadcast network, with nodes passing on commands not addressed to them to all other nodes downstream of themselves. Data sources can include packet specific extensions to the packet headers generated by them. The following fields are suggested as a mandatory part of the packet header:

- Source identifier: At every level of the tree, nodes are endowed with a unique identifier which supplants the existing upstream source id, if any processing is carried out on the packet.

- Datatype: This field allows processing entities to recognize which packets are palatable to them and to reject others.

- Data pixel descriptor: This field lays out the size and description of the smallest unit of data transfer to be one of an allowed set, which is implementation dependent.

- Streams: This field records the number of independent signal sources present in each packet.

- Packet size: The size of a packet is expressed in units of words as specified by the datatype field.

- Timestamp: This field is populated as early as possible in the data generation path and maintained across data processing. This field is generally populated by a timestamp counter running on either a reference clock or on the sampling clock itself and is traceable to the centrally maintained time standard.

This specification is efficient for real-time streaming data description with minimum overhead. For archival of processed data, a standard format which allows multiple 
binary streams to maintain their identity, like the FITS or VLBI Data Interchange Format (as proposed by the VDIF Task Force (2009)) can be used.

\subsection{Load Partitioning and Scheduling:}

No subsystem in our scheme is source synchronous, be it at the hardware or the software level. All subsystems have enough memory for a store-and-forward of several packets. This allows the processing to happen at the packet level, on a faster clock than the sampling clock of front end ADCs. It also eases the timing requirements of designs implemented in FPGAs and makes them more tolerant of clocking errors. Sequencers play an important role in our architecture, generating events on which the processing progresses. The sequencers generate necessary globally aligned events to which any action taken on the basis of commands from commodity network will get aligned. A simple example is an implementation where all processes in the peer network operate at a block level, with a periodic event signifying the need for scheduling a new process as a result of the arrival of a new block of data.

It is important to match the communication bandwidth to processing abilities at every level in the signal processing tree. More specifically, event markers generated by the sequencers should facilitate a partitioning of processing in each level into abstract transactions, where each transaction deals with the entire data collected over a convenient timeslice and the relevant data are locally available on demand. In particular, it is desirable that processing at the central commodity segment is facilitated at a cadence suited for general purpose operating system scheduling to achieve latency tolerance. For instance, to be commensurate with a housekeeping tick of 10 milliseconds in typical Linux configurations, the transaction timeslices should be several times longer.

\subsection{Choice of fusing dimension:}

Among the available axes in processing space along which the data can be partitioned and distributed to parallel processors, the time axis is often the most convenient for slicing, as individual timeslices can be considered independent. For a large network, we realize this from a hierarchical set of Data Poolers populating different levels of the processing tree, which can collate data from different sources and partition them along the time dimension at each level.

\section{An Implementation Example: Reconfiguration Plan for the Ooty Radio} Telescope

In this section, we provide an example of implementation of NSPS by giving an outline of the system being planned for modernizing the ORT (Swarup et. al, 1971). The ORT is a $506 \mathrm{~m} \mathrm{X} 30 \mathrm{~m}$ equatorially mounted cylindrical telescope with an equispaced linear array of 1056 dipoles along the focal line. Each dipole has a tuned low noise amplifier (Selvanavagam et. al, 1993) with about $40 \mathrm{MHz}$ bandwidth centered at $327 \mathrm{MHz}$, although the existing analog phasing network restricts the bandwidth to about 10 $\mathrm{MHz}$. A collaborative program for upgrading the ORT has been undertaken jointly by the Raman Research Institute (RRI) and the National Centre for Radio Astrophysics 


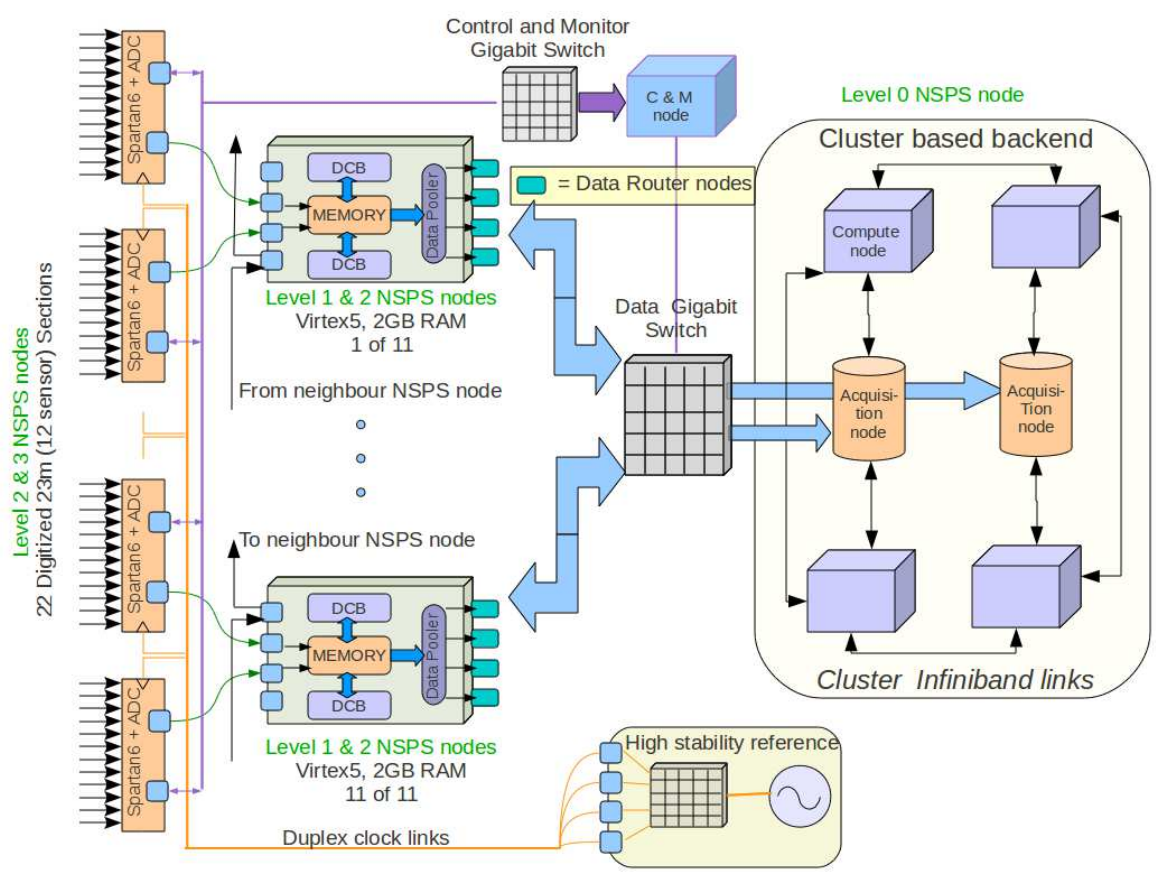

Fig. 4 Proposed NSPS implementation for configuring ORT as a 264 element programmable telescope.

(NCRA), which operates the ORT. In this program, the feed array is logically divided into 264 identical segments, where each segment represents an independent antenna element of size $1.93 \mathrm{~m} \times 30 \mathrm{~m}$. The aim of this upgrade is to reconfigure the ORT into a programmable 264-element array. When completed, the reconfigured ORT will have an instantaneous field of view of $\sim 27^{\circ}$, bandwidth of $\sim 35 \mathrm{MHz}$ and will be equipped with an NSPS-based digital receiver. Currently, prototypes have been tested for a large fraction of the custom segment of the NSPS and the analog signal conditioning subsystems. The final production and integration is expected to be completed in 2011. The digitizers are organized in 22 digitization blocks located below the reflector at a spacing of about $23 \mathrm{~m}$, where each digitization block includes a 12-channel digitizer capable of operating at $100 \mathrm{Ms} / \mathrm{s}$. All the 22 digitization blocks are connected in a star topology with a central system using a peer-to-peer network on optical fibres with multiple links operating at speeds of 2.5 Gigabits/sec from each block.

\subsection{The ORT NSPS layout:}

The proposed system consists of four hierarchical levels as illustrated in Fig. 4 where level-0 is the root node and realized by a high performance cluster with Infiniband for inter-node communication and GigE for communication with the NSPS. The high speed peer network uses the light weight Aurora protocol simplex links for data uplink, with last mile via GigE. Currently, bandwidths of upto 100MBps per GigE link into level-0 memory have been sustained. 
- Level 3: This level is composed of the distributed digitization infrastructure and is installed at the antenna base. The prime components of this level are the digitizers, the sampling clock derivation and conditioning circuitry, the first level data organizer and peer-to-peer link handler. All these components required for handling 12 sensor elements from a $23 \mathrm{~m}$ section of the ORT are implemented as a single board.

- The ADCs (dual channel AD9600) are capable of a 100Ms/s sampling for signals with frequencies upto $\sim 600 \mathrm{MHz}$. The $\sim 60 \mathrm{~dB}$ dynamic range at 10 bit resolution allows us to implement an AGC in software. More importantly, the implementation can benefit from the on-chip sampling clock conditioner, divider and duty cycle stabilizer. For instance it is possible to provide a sinewave with frequency 2-8 times the sampling clock, and use the on-chip features to convert to square-wave, enable duty-cycle stabilizer and divide by suitable integer to get the sampling clock, thus reducing the overall sampling clock jitter and hence the phase noise in the sampled data. This feature is useful in direct (harmonic) sampling of the incoming $327 \mathrm{MHz}$ RF since the Nyquist sampling interval in a band-limited RF is decided only by the bandwidth while jitter tolerance depends on the highest frequency content.

- This level has an embedded clock synthesizer and distributor for the on-board 12-channel ADC based on a reference distributed on fibre by the central high stability clock distributor. It is received using a digital fibre optic receiver. The embedded clock distributor is based on a clock buffer and distributor (LMK03020) which has an on-chip VCO and per-port delay tuning.

- The first Data processing block is implemented in a Xilinx Spartan6 (LX45T) FPGA to perform a conversion of the ADC 10 bit resolution data to $\sim 4$ bit via configurable, table-driven logic, where the look-up-table (LUT) is dynamically updated to accommodate innovative schemes of compression and segregation. For instance, let us assume that a choice among a pre-determined set of LUTs is best suited for coding/compressing the data in a set of physical packets associated with a transaction unit. Here, each table implements a different encoding of the input word to an output with lesser number of bits per word. Further, we assume that every word of each physical packet is encoded by a choice between two LUTs out of the set, to represent normal and segregated(flagged) data. For decompression by downstream nodes, the tags of these two LUTs can be accommodated in the packet header, while a one-bit selection between the two can be associated with each data word - thus achieving the dual purpose of flagging and scaling at the word level. Such a scheme can accommodate a wide range of scale factors and hence a large dynamic range within a logical packet. Suitable thresholds for packet-level choice of LUTs can be generated on the basis of integrated power over a reasonable time stretch as part of the pre-processing.

- The Data Router node buffers data from all 12 sensors in internal memory and reorganizes them to form packets containing identical time-stretches from all antenna elements. The peer-to-peer link out of each $23 \mathrm{~m}$ section which connects level-3 to level-2 is implemented using the 4 available RocketIO multi-gigabit onboard serializers on the Spartan6. Our choice of SFP for the current implementation can sustain link speeds of upto $2.6 \mathrm{Gbps}$ on single mode fibre, while we use the light-weight Aurora protocol at a wire speed of $2.5 \mathrm{Gbps}$ for communication between level 3 and level 2 . 
- Level 2: This level is implemented using an FPGA (Virtex5 LX50T) board whose on-board resources include $2 \mathrm{~GB}$ of memory and 8 multi-gigabit transceivers and expansion connectors. This board can sustain the following level-2 functionalities:

- FFT block: Here, the data processor block first decodes data from a pair of sensors, packs them into the real and imaginary parts of a 32-bit complex integer word, and implements a pipeline stage (e.g., radix-64) of a split radix FFT for all pairs of incoming channels. The processing resources are enough to handle upto 24 channels (2 level-3 entities) at the maximum sampling rate.

- The Data Pooler block operates using the large local DDR2 RAM and the interconnection with other level-2 cards to pool subsets of both local and remote level-2 data into transaction oriented packets by partitioning data along the time axis. Here, each transaction refers to the processing of a specific timeslice for the entire array.

- The Data router block collects data from the local RAM in units appropriate for transfer to each outgoing port, packetizes them and sends out selected time slices to level-1 entities. Provision for computation offloading is provided in the form of spare peer-to-peer links which can add on more level-2 cards.

- Level 1: At this level, a memory-based, NSPS to commodity network bridge is implemented. Large bursts of continuous time slices are first buffered in RAM, and then sent out over GigE as properly timestamped packets. The level implements load partitioning as configured by the root level by manipulating ethernet destination addresses of streams going into the data GigE switch. There is possibility of implementing a data processor block for the remaining 16 point FFT operation pending from the split radix FFT.

- Level 0: At the root of the NSPS tree, a medium level cluster is proposed to handle both the communication and processing requirements for forming correlations between all sensors. It is important to note that the cluster inter-node traffic is significantly reduced due to the data routing and transposition carried out using the Data Pooling nodes at the various levels. The formation of actual correlations and the calibration parameter estimation is carried out by this level. The above mentioned partitioning of the load into the 3 levels can be used to bring a subset of data from all 264 elements into one node via a quad-GigE card.

\subsection{System Control:}

The Control network is a simplex, one-way channel from a master with unique id (in the commodity segment) to the peer network through the bridge node. Thus, while data can be routed to arbitrary nodes in the cluster depending on the UDP destination addresses set during configuration, commands are accepted by the bridge node only through a privileged link from the master. This simplifies assigning privileges to operations related to starting, stopping or resetting the acquisition state machines, configuring the network routes on customized hardware switches, changing ethernet destination addresses, or changing the contents of the LUTs used in earlier nodes like the digitizers. 


\section{Discussion}

While asynchronous, packetized processing over standard networks is a relatively new concept in radio telescopes, it is being embraced enthusiastically due to the many benefits it offers to the system designer. Even among this class of telescope data processors, contemporary architectures usually have a direct link from the samplers to the central processor. Some operations like a digital filter bank or FFT are carried out remotely, while others like cross-correlation is done centrally. The memory-rich architectures of modern FPGAs help in distributing computing to remote nodes and enables buffering to allow multiple passes on the streaming data. As the data volume grows, e.g., in the central pooling stations, the processing can be supported by large off-chip memory using commercial memory modules, routinely supported by modern FPGAs. This provides substantial enhancement to buffering for transaction level operations and data partitioning. A peak data rate of $100 \mathrm{Ms} / \mathrm{s}$ x 4 bits for 264 elements for the ORT would correspond to about $13.2 \mathrm{~GB} / \mathrm{s}$ for which the level- 1 buffering of 22 GB in 11 Virtex- 5 cards shown in Fig. 4 is comfortable to sustain transactions of upto a fraction of a second duration. The use of standard software stacks also allows us to leverage the various high performance modes being worked upon by system optimizers, e.g., the zero copy mechanism on Linux.

Another challenging problem with large arrays is the so-called corner turning problem, which refers to the transposition of the input signal matrix needed to achieve the all-to-all communication necessary for correlation. Earlier approaches have looked at either commercial switches or entirely customized switches for routing data (Lutomirski et. al, 2010). We break this problem down into levels, and apply a hybrid of commercial as well as custom routing. The Data Pooler element is utilized to implement a memory based switch, while the COTS (GigE) network controller manages another level of redirection by manipulating UDP destination addresses.

\section{Conclusions}

We have presented a packetized, heterogeneous and distributed signal processing architecture for radio interferometric signal processing which elevates the network to a core system component. The architecture addresses some of the core issues pertaining to interferometric signal processing. We visualize this problem as that of an appropriate workload creation and scheduled dispatch to matched processors over a data flow tree. Here, the leaf nodes are sources of data, with data processors handling a managed slice of the processing at the intermediate nodes of this tree. We emphasize the use of COTS components, both hardware and software, for rapid deployment, ease of maintenance, and lowering the cost of the architecture implementation.

The goal of realizing a programmable telescope with NSPS is facilitated by defining rigid interfaces between both hardware and software components. This can allow exchange of a variety of data with varying communication and computing requirements between levels in the network. Most of the individual nodes in the NSPS can change the nature of their processing within the limits specified by their designed personality and available resources at the node. This allows offloading of computing requirements in a hierarchical manner up the NSPS tree, trading off implementation time with hardware capability of an application mode. Due to the rigidity of interfacing protocols as well as the standardized networks making up the system, we can comfortably add nodes 
which can tap into the NSPS in order to carry out a different processing chain. Data duplication, if required, can be carried out by COTS components (e.g., by switches operating in broadcast mode) thus reducing development load.

We have presented an outline of the NSPS implementation being planned for configuring the ORT as a programmable 264 element telescope. Our architecture is optimally tuned to service the needs of medium sized arrays. We advocate full software processing for smaller arrays, with an increasing factor of hardware offload as the array size grows. This approach has being taken by us in building a 44 element demonstrator as a precursor to the receiver for the full 264 element ORT array. This receiver exploits all NSPS aspects we have dwelt on, and is in an advanced stage of completion.

Acknowledgements The 44-element demonstrator for the ORT is being built as a collaborative effort between Radio Astronomy Laboratory at Raman Research Institute and the observatory staff at the Ooty Radio Telescope. Many of the ideas presented here evolved during the trials of demonstrator subsystems for which we specially thank colleagues both at RRI and ORT. CRS would like to thank Madan Rao and Dwarakanath whose comments have helped improving the manuscript. We thank the anonymous referee whose comments have been very helpful in improving the clarity of presentation in the manuscript

\section{References}

Cover T.M. and Thomas J. A.: Elements of Information Theory, Second Edition, Chapter 15, Wiley (2006)

Klein, L. A.: Sensor and, Data Fusion: A Tool for Information Assessment and Decision Making, SPIE Press (2004)

Lutomirski et. al.: Solving the Corner-Turning Problem for Large Interferometers, arXiv:0910.1351v1 (2010)

Parsons, A., et al.:Digital Instrumentation for Radio Astronomy Community, arXiv: 0914.1181v1 (2009)

Roy, J., et. al.: A Real-Time Software Backend for the GMRT. Exp. Astron. 28, 25-60 (2010)

Selvanayagam, A. J., et. al.: Sensitivity Boost to the Ooty Radio Telescope: A New Phased Array of 1056 dipoles with 1056 Low Noise Amplifiers. IETE Tech. Rev., 10, 4, 333-339 (1993)

Swarup, G., et al.: Large Steerable Radio Telescope at Ootacamund, India. Nat. Phys. Sci. 230, 185-188 (1971)

Thompson, A.R., Moran, J.M. and Swenson, G.W.: Interferometry and Synthesis in Radio Astronomy, Kreiger Publishing Company (1994)

Zurek, W.H.: Thermodynamic cost of computation, algorithmic complexity and the information metric, Nature, 341, 119 (1989). 